Research Article

\title{
On Numerical Thermal Transport Analysis of Three-Dimensional Bioconvective Nanofluid Flow
}

\author{
Jifeng Cui $\mathbb{D}^{1},{ }^{1}$ Shahzad Munir, ${ }^{2}$ Umer Farooq $\mathbb{D}^{2},{ }^{2}$ Mohammed Elamin Ahmed Rabie, ${ }^{3}$ \\ Taseer Muhammad $\mathbb{D}^{4},{ }^{4}$ and Raheela $\operatorname{Razzaq}^{2}$ \\ ${ }^{1}$ College of Science, Inner Mongolia University of Technology, Hohhot 010051, China \\ ${ }^{2}$ Department of Mathematics, COMSATS University Islamabad, Islamabad Campus, Islamabad 44000, Chak Shehzad, Pakistan \\ ${ }^{3}$ Department of Mathematics, Faculty of Science and Humanities, Shaqra University, Afif, Saudi Arabia \\ ${ }^{4}$ Department of Mathematics, College of Sciences, King Khalid University, Abha 61413, Saudi Arabia
}

Correspondence should be addressed to Jifeng Cui; cjf@imut.edu.cn and Taseer Muhammad; taseer_qau@yahoo.com

Received 29 May 2021; Accepted 19 July 2021; Published 28 July 2021

Academic Editor: M. M. Bhatti

Copyright (C) 2021 Jifeng Cui et al. This is an open access article distributed under the Creative Commons Attribution License, which permits unrestricted use, distribution, and reproduction in any medium, provided the original work is properly cited.

In this paper, a numerical study is presented for the 3D mathematical model of bioconvective boundary layer flow having nanoparticles and motile microorganisms on a curved sheet under isothermal conditions. Using an appropriate choice of similarity transformations, the problem reduces to coupled ordinary nonlinear equations, and this system is then treated with bvp4c (a MATLAB-based solver) to get the desired solution with good accuracy. The repercussion of distinct important dimensionless numbers such as thermophoresis, buoyancy ratio, Lewis number, and Brownian number on the velocity, temperature, and volume fraction of nanoparticles is presented graphically and is discussed in context with their importance on flow dynamics. Moreover, the physical impact of various parameters on motile microorganism density, the local Sherwood number, the local Nusselt number, and the local skin friction coefficients is analyzed and presented in tables. Qualitative analysis also reveals that the Brownian motion parameter, Peclet number, and Schmidt number have an inverse impact on the density microorganisms.

\section{Introduction}

A fluid containing nanoparticles in a basic fluid called nanofluid. The diameter of nanoparticles varies from 1 to $100 \mathrm{~nm}$ and is generally made of nitrides, carbides, oxides, metals, or nonmetals. These have an ability to improve heat transfer properties such as viscosity, thermal conductivity, and diffusivity in fluids, for example, ethylene glycol, oils, and water. Choi and Eastman [1] proposed "nanofluids" as a new innovative class of heat transfer fluids to enhance high thermal conductivities. Their theoretical study on these nanofluids resulted in reduction in heat exchanger pumping power. Nanofluids have broad range of implementations in mechanical, chemical engineering, civil, thermal energy storage devices, ground water structure, electronic cooling, heat loss from piping, boilers, crude oil extraction, etc.
Detailed discussion related to nanofluids can be found in [2-6]. Using the Buongiorno [7] model of nanofluids, Kuznetsov and Nield [8] carried out a study to analyze natural convection in nanofluid flow over a vertical smooth surface. Bachok et al. [9] inspected heat transfer effects for a steady 3D stagnation flow of nanofluids to explore the impacts of the ratio of gradient of velocities and the nanoparticles volume fraction. In another study, Bachok et al. [10] examined nanofluid flow on top of an expanding and contracting plate in two dimensions. Alsaedi et al. [11] implored the significance of heat absorption and generation on a permeable and linear stretching plate of the nanofluid stagnation point flow. Razzaq et al. [12] studied the magnetized flow of non-Newtonian Maxwell nanofluid over an exponentially stretching sheet by utilizing the local nonsimilarity method. 
Microorganism particles have been widely utilized to construct commercial and industrial material such as bio fuel derived from waste/biomass, fertilizers, ethanol, and many more. These products are also employed in dairy products, steroid production, and water treatment plants. These microorganisms are a cause of a renewable energy origin named as hydrogen gas and biodiesel. That is the reason of research need to explore the mass transmission and swimming path properties of the microorganisms so that usage of the organisms might be more appealing and profitable for the welfare of the human being. Bioconvection is evolution of distinct kind of random fluid design at the microscopic label due to the spontaneous swimming of selfpropelled microorganisms which are adjacent in the water and in the other liquids denser than water. Bioconvection have wide applications in the bio-microsystems, biomedical sciences, biotechnology, gas-containing sedimentary basin, and modeling oil [13]. A quantitative explanation of bioconvection flow patterns by using Rayleigh-Taylor instability is presented by Milton and Plesset [14]. Childress et al. [15] and Levandowsky et al. [16] established the first mathematical model for bioconvective flows in which they take stress-free and rigid upper boundary containing a suspension in which entirely upward swimming cells existed. Pedley et al. [17] described a gyrotactic bioconvection continuum model using a uniform diffusivity for finite and infinite depth of layers. Furthermore, Pedley and Kessler [18] extended this model in an entirely consistent mode. Bees [19] investigated the stochastic nature of the microorganism swimming direction using surface spherical harmonics. Bees and Hill [20] carried out bioconvection analysis in a realistic geometry by developing a systematic model that primarily predict and calculate the irregular swimming motion in an analytical way. Ghorai et al. [21-23] used finite-difference technique to observe the formation and solidity of gyrotactic plumes in two-dimensional large, thin chambers for periodic sidewalls and stress-free sidewalls. Furthermore, Ghorai and Hill [24] investigated the stability and structure of plume in a deep cell, using the gyrotactic bioconvection model equations of Pedley et al. [17]. Kuznetsov investigated the mobility of the suspension of moveable microorganisms [25-27]. Geng et al. analyzed the composition of nanoparticles, and further, they concluded that this addition makes the nanofluid model more stable [28-30]. Lately, Zaimi et al. [31] scrutinized the addition of gyrotactic microorganisms on the heat changes for stagnation flow of nanofluids towards an expanding/contracting smooth medium.

The 3D boundary layer flow on an isothermal medium is the topic of immense interest in recent past. Howarth [32] and Davey and Schofield [33] did the pioneer work by providing the first 3D boundary layer solutions of stagnation point flow. Poots [34] expressed the equation of the boundary layer on a curved isothermal medium for the $3 \mathrm{D}$ free convection flow. Slaouti et al. [35] analyzed a threedimensional heated body with unsteady and free convection effects to describe the boundary layer properties of the fluid flow. Harris et al. [36] identified and studied the evolution of boundary layer for a compressible fluid over a cylindrical object. Xu et al. [37] provided HAM-based series solutions of 3D free convection flow on a curved smooth surface with isothermal properties. Singh et al. [38] continued the research of $\mathrm{Xu}$ et al. [37] by considering the transfer effects of mass. Admon et al. [39] worked on three-dimensional unsteady boundary layer stagnation flow with free convection effects for an ambient fluid.

In this study, we have explored by considering the gyrotactic motile microorganisms in the flow model of Farooq and $\mathrm{Xu}$ [40] and assumed viscous, steady, incompressible, laminar, and free convection nanofluid flow with gyrotactic motile microorganisms and nanoparticles. The governing Navier-Stokes, energy conservation, concentration of nanoparticle, and microorganisms' density equations are changed into nonlinear ODEs along with the boundary conditions by applying an appropriate similarity transformation. The system of ODEs is numerically simulated by using a MATLAB routine bvp4c and examined the influences of distinct physical parameters on microorganisms' density, velocity, nanoparticles concentration, and temperature. The influence of that physical parameter on local microorganism's density, local Sherwood number, and local Nusselt number are also examined to comprehend the overall flow dynamics and importance of physical parameters on flow physics.

\section{Mathematical Model}

Consider a 3D viscous, steady, laminar, and incompressible free convection nanofluid flow with gyrotactic motile microorganisms and nanoparticles. In Figure 1, the schematic diagram of the physical fluid flow is presented. The $3 \mathrm{D}$ rectangular system along with three velocity components is shown, where $x$ - and $y$-axes are along the body surface, and the flow is taken along $z$-axis, which is orthogonal to the surface of the body.

$C_{w}$ is the concentration of nanoparticle, $T_{w}$ is temperature, and $N_{w}$ is the microorganisms density. For the case of ambient fluid these are $C_{\infty}, T_{\infty}$, and $N_{\infty}$, respectively, and $g$ is the gravitational force. Furthermore, it is supposed that the concentration of nanoparticles is not concentrated and uses Oberbeck-Boussinesq approximation. Based on the abovementioned assumptions and according to Buongiorno [7] and Kuznetsov and Nield [8] models, the governing equations in the scalar form are 


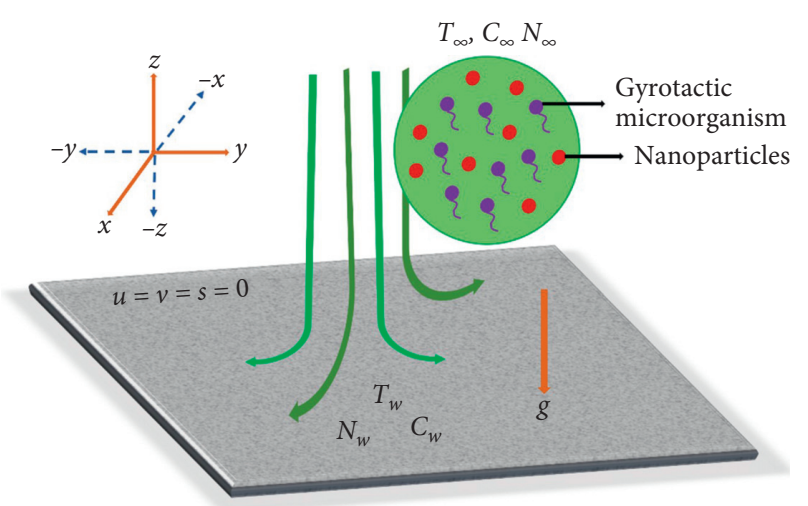

Figure 1: Physical flow configuration.

$$
\begin{aligned}
\frac{\partial u}{\partial x}+\frac{\partial v}{\partial y}+\frac{\partial s}{\partial z}= & 0 \\
u \frac{\partial u}{\partial x}+v \frac{\partial u}{\partial y}+s \frac{\partial u}{\partial z}= & -\frac{1}{\rho f_{\infty}} \frac{\partial p}{\partial x}+v\left(\frac{\partial^{2} u}{\partial x^{2}}+\frac{\partial^{2} u}{\partial y^{2}}+\frac{\partial^{2} u}{\partial z^{2}}\right) \\
& -\left[\frac{\rho_{p}-\rho f_{\infty}}{\rho f_{\infty}}\left(C-C_{\infty}\right)-\frac{\rho_{m}-\rho f_{\infty}}{\rho f_{\infty}}(\gamma)\left(N-N_{\infty}\right)-\left(1-C_{\infty}\right) \beta\left(T-T_{\infty}\right)\right] g a x, \\
u \frac{\partial u}{\partial x}+v \frac{\partial u}{\partial y}+s \frac{\partial u}{\partial z}= & -\frac{1}{\rho f_{\infty}} \frac{\partial p}{\partial y}+v\left(\frac{\partial^{2} v}{\partial x^{2}}+\frac{\partial^{2} v}{\partial y^{2}}+\frac{\partial^{2} v}{\partial z^{2}}\right) \\
& -\left[\frac{\rho_{p}-\rho f_{\infty}}{\rho f_{\infty}}\left(C-C_{\infty}\right)-\frac{\rho_{m}-\rho f_{\infty}}{\rho f_{\infty}}(\gamma)\left(N-N_{\infty}\right)-\left(1-C_{\infty}\right) \beta\left(T-T_{\infty}\right)\right] g a y, \\
u \frac{\partial T}{\partial x}+v \frac{\partial T}{\partial y}+s \frac{\partial T}{\partial z}= & \alpha\left(\frac{\partial^{2} T}{\partial x^{2}}+\frac{\partial^{2} T}{\partial y^{2}}+\frac{\partial^{2} T}{\partial z^{2}}\right) \\
& +\tau\left[D_{B}\left(\frac{\partial C}{\partial x} \frac{\partial T}{\partial x}+\frac{\partial C}{\partial y} \frac{\partial T}{\partial y}+\frac{\partial C}{\partial z} \frac{\partial T}{\partial z}\right)+\frac{D_{T}}{T_{\infty}}\left\{\left(\frac{\partial T}{\partial x}\right)^{2}+\left(\frac{\partial T}{\partial y}\right)^{2}+\left(\frac{\partial T}{\partial z}\right)^{2}\right\}\right], \\
u \frac{\partial C}{\partial x}+v \frac{\partial C}{\partial y}+s \frac{\partial C}{\partial z}= & D_{B}\left(\frac{\partial^{2} C}{\partial x^{2}}+\frac{\partial^{2} C}{\partial y^{2}}+\frac{\partial^{2} C}{\partial z^{2}}\right)+\frac{D_{T}}{T_{\infty}}\left(\frac{\partial^{2} T}{\partial x^{2}}+\frac{\partial^{2} T}{\partial y^{2}}+\frac{\partial^{2} T}{\partial z^{2}}\right), \\
\frac{\partial N}{\partial x}+v \frac{\partial N}{\partial y}+s \frac{\partial}{\partial z}+\frac{\partial}{\partial z}(N \tilde{v})= & D_{n}\left(\frac{\partial^{2} N}{\partial x^{2}}+\frac{\partial^{2} N}{\partial y^{2}}+\frac{\partial^{2} N}{\partial z^{2}}\right) .
\end{aligned}
$$

Here, the velocity component $u$ is along $x$-axis, $v$ is along $y$-axis, and $s$ is along $z$-axis. $v$ is the fluid kinematic viscosity and $\widetilde{v}$ is the standard swim velocity of the gyrotactic microorganisms defined as follows:

$$
\widetilde{v}=\left(\frac{\tilde{b} W_{c}}{\Delta C}\right) \frac{\partial C}{\partial z} .
$$

The boundary conditions for equations (1)-(6) are

$$
\begin{gathered}
u=v=s=0, T=T_{w}, C=C_{w}, N=N_{w} \text {, at } z=0, u \longrightarrow 0, v \longrightarrow 0 \\
\cdot C \longrightarrow C_{\infty}, T \longrightarrow T_{\infty}, N \longrightarrow 0, \text { as } z \longrightarrow \infty
\end{gathered}
$$


The following similarity transformations are introduced:

$$
\begin{aligned}
\eta & =\mathrm{Gr}^{1 / 4} a z, \\
u & =v a^{2} \mathrm{Gr}^{1 / 2} x f^{\prime}, \\
v & =v a^{2} \mathrm{Gr}^{1 / 2} c y h^{\prime}, \\
s & =-v a \mathrm{Gr}^{1 / 4}(f+\mathrm{ch}), \\
\theta(\eta) & =\frac{T-T_{\infty}}{T_{w}-T_{\infty}}, \\
\phi(\eta) & =\frac{C-C_{\infty}}{C_{w}-C_{\infty}}, \\
w(\eta) & =\frac{N-N_{\infty}}{N_{w}-N_{\infty}} .
\end{aligned}
$$

Substituting (9) into equations (1)-(6) and (8), we get ODE's (10)-(14) and boundary conditions (15) as follows:

$$
\begin{aligned}
& f^{\prime \prime \prime}+(f+\mathrm{ch}) f^{\prime \prime}-\left(f^{\prime}\right)^{2}+\theta-\left(\frac{N_{r}}{P_{r} G_{r}}\right) \phi-\left(\frac{R_{b}}{P_{r} G_{r}}\right) w=0, \\
& h^{\prime \prime \prime}+(f+\mathrm{ch}) h^{\prime \prime}-c\left(f^{\prime}\right)^{2}+\theta-\left(\frac{N_{r}}{P_{r} G_{r}}\right) \phi-\left(\frac{R_{b}}{P_{r} G_{r}}\right) w=0 \text {, } \\
& \frac{1}{\operatorname{Pr}} \theta^{\prime \prime}+(f+\mathrm{ch}) \theta^{\prime}-\frac{N_{b}}{\operatorname{Pr}} \theta^{\prime} \phi^{\prime}+\frac{N_{t}}{\operatorname{Pr}}\left(\theta^{\prime}\right)^{2}=0, \\
& \phi^{\prime \prime}+\left(\frac{N_{t}}{N_{b}}\right) \theta^{\prime \prime}+\operatorname{LePr}(f+\mathrm{ch}) \phi^{\prime}=0 \\
& w^{\prime \prime}+\operatorname{Sc}(f+\mathrm{ch}) w^{\prime}-\operatorname{Pe}\left(w^{\prime} \phi^{\prime}+(w+\sigma) \phi^{\prime \prime}\right)=0, \\
& f(0)=0 \text {, } \\
& f^{\prime}(0)=1 \text {, } \\
& f^{\prime}(\infty)=0 \text {, } \\
& h(0)=1 \text {, } \\
& h(\infty)=0 \text {, } \\
& h^{\prime}(\infty)=0 \text {, } \\
& \theta(0)=1 \text {, } \\
& \theta(\infty)=0 \text {, } \\
& \phi(0)=1 \text {, } \\
& \phi(\infty)=0 \text {, } \\
& w(0)=1 \text {. }
\end{aligned}
$$

In the equation (16), nondimensional physical parameters, mixed convection parameter $\left(R_{b}\right)$, Prandtl number $(\operatorname{Pr})$, Brownian parameter $\left(N_{b}\right)$, the buoyancy parameter $\left(N_{r}\right)$, Schmidt number $(\mathrm{Sc})$, the thermophoresis parameter $\left(N_{t}\right)$, microoganisms' concentration difference parameter $(\sigma)$, Peclet number $(\mathrm{Pe})$, Lewis number (Le), and Grashof number (Gr) are defined by

$$
\begin{aligned}
N_{b} & =\frac{\tau D_{B}\left(C_{w}-C_{\infty}\right)}{v}, \\
N_{t} & =\frac{\tau D_{T}\left(T_{w}-T_{\infty}\right)}{T_{\infty} \nu}, \\
N_{r} & =\frac{\left(\rho_{p}-\rho_{f}\right)\left(C_{w}-C_{\infty}\right)}{\rho_{f} \beta\left(1-C_{\infty}\right)\left(T_{w}-T_{\infty}\right)}, \\
R_{b} & =\frac{g(\gamma)\left(\rho_{p}-\rho f_{\infty}\right)\left(N_{w}-N_{\infty}\right)}{\mu \alpha a^{3}}, \\
\mathrm{Gr} & =\frac{g \beta \rho_{f}\left(1-C_{\infty}\right) a^{3}\left(T_{w}-T_{\infty}\right)}{v^{2}}, \\
\sigma & =\frac{N_{\infty}}{N_{w}-N_{\infty}}, \\
\mathrm{Le} & =\frac{\alpha}{D_{B}}, \\
\mathrm{Sc} & =\frac{v}{D_{n}}, \\
\operatorname{Pr} & =\frac{b w_{c}}{D_{n}}, \\
& =\frac{v}{a},
\end{aligned}
$$

where $\alpha$ is the thermal diffusivity. To physically describe the flow dynamics, the important flow quantities such as the skin friction $C_{f x}$ and $C_{f y}$, the Nusselt number $\mathrm{Nu}_{x}$ and $\mathrm{Nu}_{y}$, the microorganisms' density $Q_{n x}$ and $Q_{n y}$, and wall mass flux $Q_{m x}$ and $Q_{m y}$ in $x$ - and $y$-directions are defined as follows:

$$
\begin{aligned}
\mathrm{Nu}_{x} & =\frac{x q_{I}}{K\left(T_{w}-T_{\infty}\right)}, \\
\mathrm{Nu}_{y} & =\frac{y q_{I}}{K\left(T_{w}-T_{\infty}\right)}, \\
C_{f x} & =\frac{\tau_{w x}}{\rho u_{x}^{2}} \\
C_{f y} & =\frac{\tau_{w y}}{\rho u_{x}^{2}}, \\
Q_{m x} & =\frac{x q_{m}}{D_{B}\left(C_{w-} C_{\infty}\right)}, \\
Q_{m y} & =\frac{y q_{m}}{D_{B}\left(C_{w-} C_{\infty}\right)}, \\
Q_{n x} & =\frac{x q_{n}}{D_{n}\left(N_{w-} N_{\infty}\right)}, \\
Q_{m x} & =\frac{y q_{n}}{D_{n}\left(N_{w-} N_{\infty}\right)},
\end{aligned}
$$


where

$$
\begin{aligned}
u_{x} & =v a^{2} \mathrm{Gr}^{1 / 2} x, \\
u_{y} & =v a^{2} \mathrm{Gr}^{1 / 2} c y, \\
\tau_{w x} & =\mu\left(\frac{\partial u}{\partial z}\right)_{z=0}, \\
\tau_{w y} & =\mu\left(\frac{\partial v}{\partial z}\right)_{z=0}, \\
q_{w} & =-k\left(\frac{\partial T}{\partial z}\right)_{z=0}, \\
q_{m} & =-D_{B}\left(\frac{\partial C}{\partial z}\right)_{z=0}, \\
q_{n} & =-D_{n}\left(\frac{\partial N}{\partial z}\right)_{y=0} .
\end{aligned}
$$

Substituting (9) in (17), we obtain

$$
\begin{aligned}
\operatorname{Re}_{x}^{1 / 2} C_{f x} & =f^{\prime \prime}(0), \\
\operatorname{Re}_{y}^{1 / 2} C_{f y} & =h^{\prime \prime}(0), \\
\operatorname{Re}_{x}^{(-1 / 2)} \mathrm{Nu}_{x} & =\operatorname{Re}_{y}^{-,(1 / 2)} \mathrm{Nu}_{y}=-\theta^{\prime}(0), \\
\operatorname{Re}_{x}^{(-1 / 2)} Q_{m x} & =\operatorname{Re}_{y}^{-1 / 2} Q_{m y}=-\phi^{\prime}(0), \\
\operatorname{Re}_{x}^{(-1 / 2)} Q_{n x} & =\operatorname{Re}_{y}^{-1 / 2} Q_{n y}=-w^{\prime}(0) .
\end{aligned}
$$

Here, the Reynolds number $\operatorname{Re}_{x}=u_{x} x / \nu$ is along $x$-coordinate and $\operatorname{Re}_{y}=u_{y} x / \nu$ is along $y$-coordinates.

\section{Results and Discussion}

The system of ODEs (10)-(14) along with the boundary condition (15) is coupled and nonlinear. To solve this system numerically, we used MATLAB-based routine bvp4c. For this purpose, we convert the nonlinear ODEs into 1st order differential equations. For this, we suppose

$$
\begin{aligned}
f & =f_{1}, \\
f^{\prime} & =f_{2}, \\
f^{\prime \prime} & =f_{3}, \\
f^{\prime \prime \prime} & =f_{3}^{\prime}, \\
h & =f_{4}, \\
h^{\prime} & =f_{5}, \\
h^{\prime \prime} & =f_{6}, \\
h^{\prime \prime \prime} & =f_{6}^{\prime}, \\
\theta & =f_{7}, \\
\theta^{\prime} & =f_{8}, \\
\theta^{\prime \prime} & =f_{8}^{\prime}, \\
\phi & =f_{9}, \\
\phi^{\prime} & =f_{10}, \\
\phi^{\prime \prime} & =f_{10}^{\prime}, \\
w & =f_{11}, \\
w^{\prime} & =f_{12}, \\
w^{\prime \prime} & =f_{12}^{\prime} .
\end{aligned}
$$

Replacing the above assumption into equations (10)-(14), we obtain

$$
\begin{aligned}
& f^{\prime \prime \prime}=f_{3}^{\prime}=f_{2}^{2}-\left(f_{1}+c f_{4}\right) f_{3}-f_{7}+\left(\frac{N_{r}}{P_{r} G_{r}}\right) f_{9}+\left(\frac{R_{b}}{P_{r} G_{r}}\right) f_{11}, \\
& h^{\prime \prime \prime}=f_{6}^{\prime}=c f_{5}^{2}-f_{7}-\left(f_{1}+c f_{4}\right) f_{6}+\left(\frac{N_{r}}{P_{r} G_{r}}\right) f_{9}+\left(\frac{R_{b}}{P_{r} G_{r}}\right) f_{11}, \\
& \theta^{\prime \prime}=f_{8}^{\prime}=\operatorname{Pr}\left[-\left(f_{1}+c f_{4}\right) f_{8}-\frac{N_{b}}{\operatorname{Pr}} f_{8} f_{10}-\frac{N_{t}}{\operatorname{Pr}} f_{8}^{2}\right], \\
& \phi^{\prime \prime}=f_{10}^{\prime}=-\operatorname{LePr}\left(f_{1}+c f_{4}\right) f_{10}-\frac{N_{t}}{\operatorname{Pr}} f_{8}^{\prime}, \\
& w^{\prime \prime}=f_{12}^{\prime}=\operatorname{Pe}\left(f_{12} f_{10}+\left(f_{11}+\sigma\right) f_{10}^{\prime}\right)-\operatorname{Sc}\left(f_{1}+c f_{4}\right) f_{11},
\end{aligned}
$$

which subjected to the given conditions:

$$
\begin{aligned}
f_{1}(0) & =1, \\
f_{2}(0) & =0, \\
f_{2}(\infty) & =0, \\
f_{4}(0) & =0, \\
f_{5}(0) & =0, \\
f_{5}(\infty) & =0, \\
f_{7}(0) & =1, \\
f_{7}(\infty) & =0, \\
f_{9}(0) & =1, \\
f_{9}(\infty) & =0, \\
f_{11}(0) & =1, \\
f_{11}(\infty) & =0 .
\end{aligned}
$$

Due to the abovementioned efforts, the ODEs (10)-(14) with corresponding conditions (15) are numerically solved by MATLAB package bvp4c. It is noticed that the results obtained from bvp4c have an error less than $1 \times 10^{-8}$.

In the following discussion, we have analyzed the influences of different physical numbers such as $N_{t}$, Le, $N_{b}, \mathrm{Sc}, \mathrm{Pe}$, and $N_{r}$ on the temperature, velocity, microorganisms' density, and nanoparticles' concentration graphically. Similarly, how the parameters influenced physically designed measures such as skin friction, Nusselt number, motile microorganism's density, and wall mass flux are also discussed in detail and presented in tables.

The buoyancy coefficient $N_{r}$ effects on fluid velocity are described in Figures 2 and 3. The buoyancy parameter increases due to increase in the density difference; therefore, fluid flow decreases; however, the difference in density becomes zero when there is no buoyancy resulting in maximum fluid flow. Figures 4 and 5 present the behaviour of dimensionless concentration and temperature profiles for various values of Le for buoyancy backed flow $T_{w}>T_{\infty}$. The temperature is maximum on the wall, but, as soon as we walk away from the wall, the temperature starts to decrease, and it approaches to the free stream temperature asymptotically. 


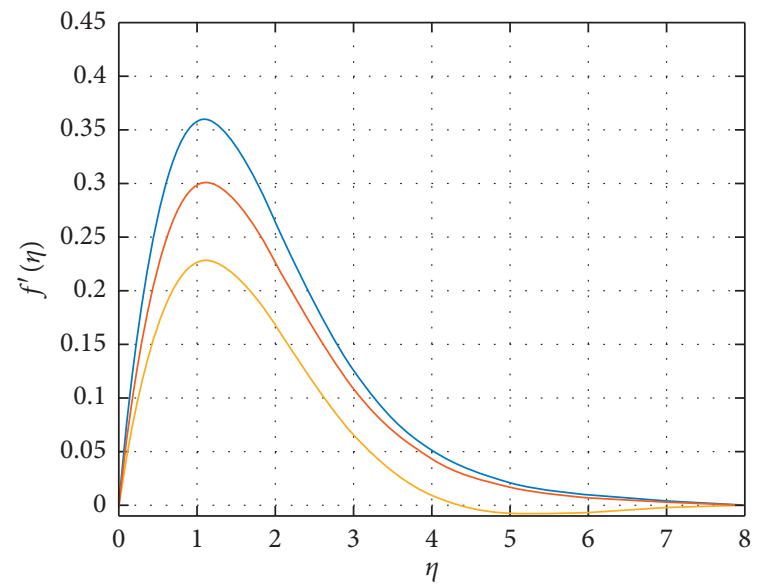

$$
\begin{aligned}
\mathrm{Nr} & =0 \\
\mathrm{Nr} & =0.2 \\
\mathrm{Nr} & =0.4
\end{aligned}
$$

Figure 2: Plot of $f^{\prime}(\eta)$ for variation in $N_{r}$ when $\mathrm{Pr}=\mathrm{Le}=\mathrm{Gr}=\mathrm{Sc}=\mathrm{Pe}=1.0, c=0.5$, and $N_{t}=N_{b}=0.1$.

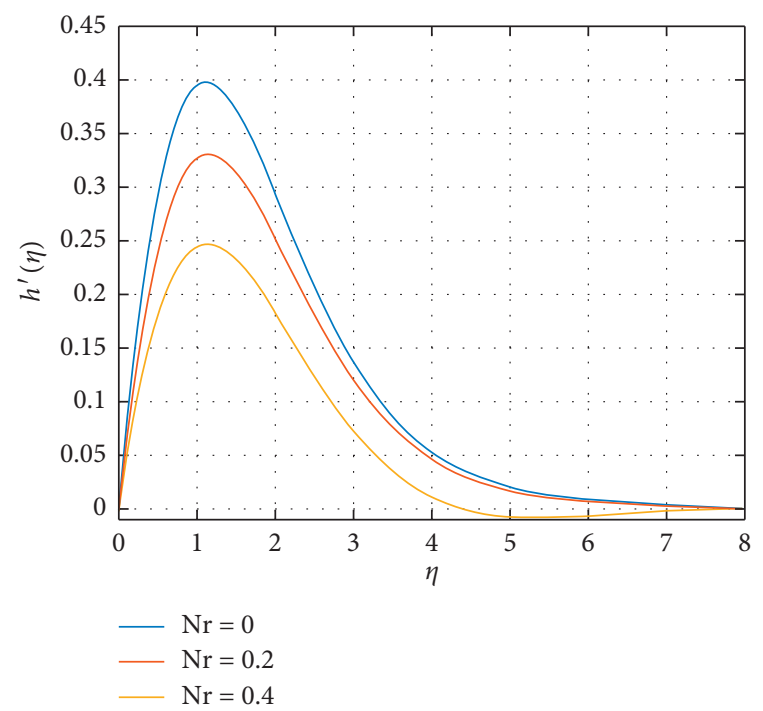

Figure 3: Plot of $h^{\prime}(\eta)$ for variation in $N_{r}$ when $\mathrm{Pr}=\mathrm{Le}=\mathrm{Gr}=\mathrm{Sc}=\mathrm{Pe}=1.0, c=0.5$, and $N_{t}=N_{b}=0.1$.

Note that an inverse relation is found between concentration and temperature profiles against Lewis number, as they decrease for high Lewis number, Le. That is why, the boundary layer thickness reduces for high Lewis number, but the impact of Le on concentration profile is more noticeable. The mass diffusivity decreases for high Lewis number, resulting in low transfer of mass across the fluid.

Figures 6 and 7 describe the thermophoresis $N_{t}$ impact on nanoparticles' concentration profile and temperature profile. An enhancement in the nanoparticles concentration and temperature graph of the fluid is observed for high values of $N_{t}$; therefore, the nanoparticles increase the mass and heat transmission. It is also remarked that $N_{t}$ effectively influences the concentration portray. It can be observed that the negative value of $N_{t}$ specifies a hot surface,

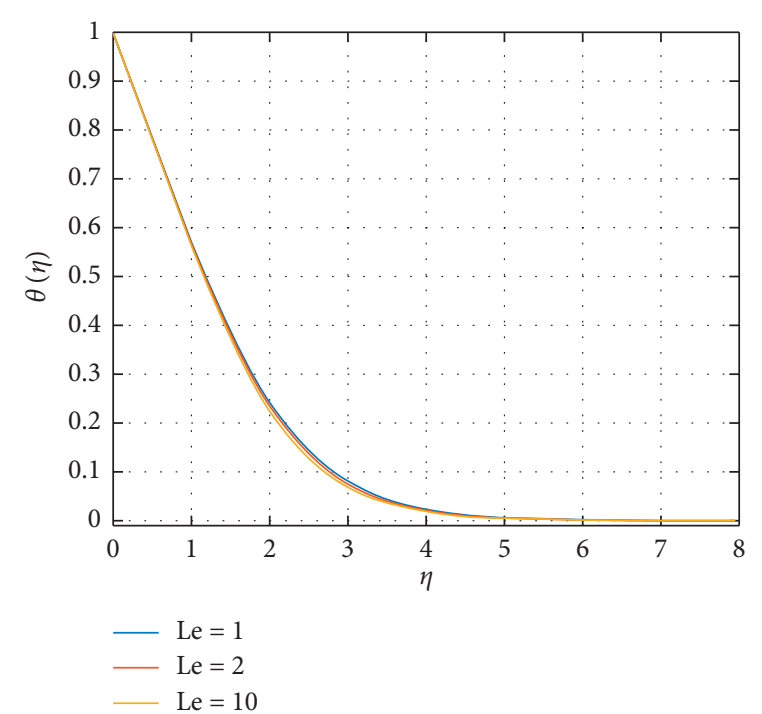

Figure 4: Plot of $\theta(\eta)$ for variation in Le when $\mathrm{Pr}=\mathrm{Gr}=\mathrm{Sc}=\mathrm{Pe}=1.0, c=0.5$, and $N_{t}=N_{r}=N_{b}=0.1$.

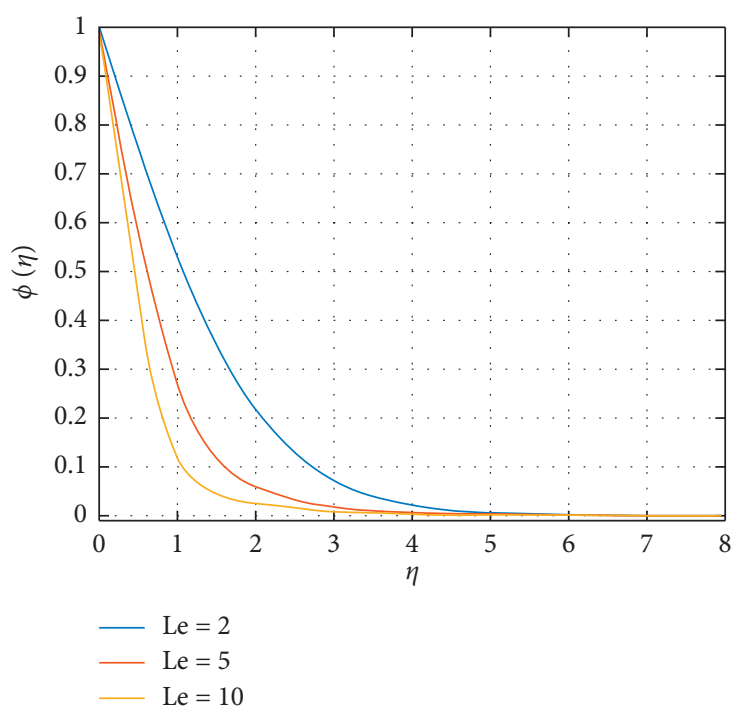

Figure 5: Plot of $\phi(\eta)$ for variation in Le when $\mathrm{Pr}=\mathrm{Gr}=\mathrm{Sc}=\mathrm{Pe}=1.0, c=0.5$, and $N_{t}=N_{b}=N_{r}=0.1$.

but positive values of $N_{t}$ specifies a cold surface. In case of the warm surface, $N_{t}$ declines the nanoparticle volume fraction boundary layer apart from the exterior since a hot top repulse the submicron-sized particles from it, thereby establishing a comparatively particle-free film close to the surface. As an outcome, the thermal, momentum, and diffusion boundary profiles are organized just outside. Specifically, the impact of enhancing $N_{t}$ is restricted to moderately declining the wall slope of the nanoparticle volume fraction portray but expanding the nanoparticle volume fraction. It is justified for smaller Lewis number Le for which the Brownian diffusion $N_{b}$ impacts are greater analogous to the convection influences. Although, for higher Le, the diffusion impact is minimal than convection impacts; hence, $N_{t}$ is expected to change the nanoparticle 


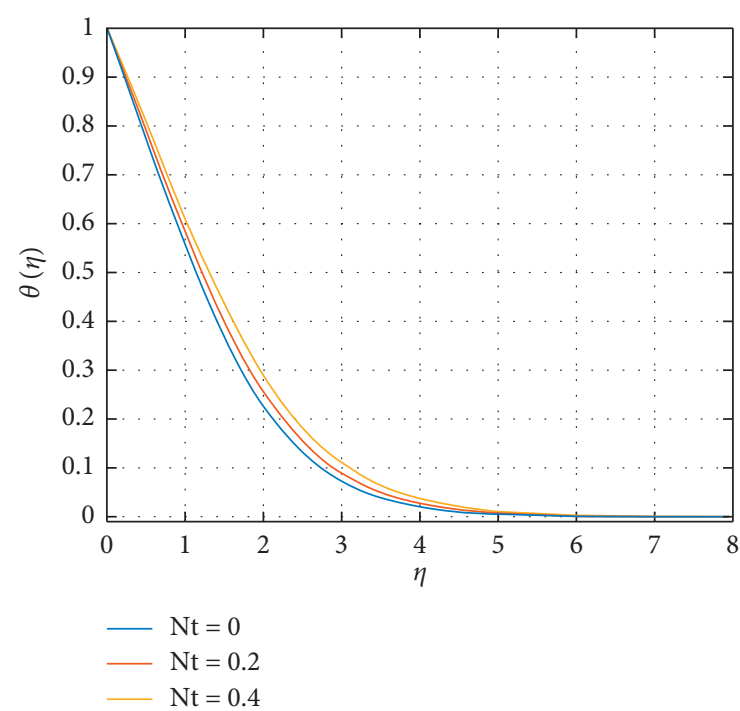

Figure 6: Plot of $\theta(\eta)$ for variation in $N_{t}$ when $\mathrm{Pr}=\mathrm{Le}=\mathrm{Gr}=\mathrm{Sc}=\mathrm{Pe}=1.0, c=0.5$, and $N_{r}=N_{b}=0.1$.

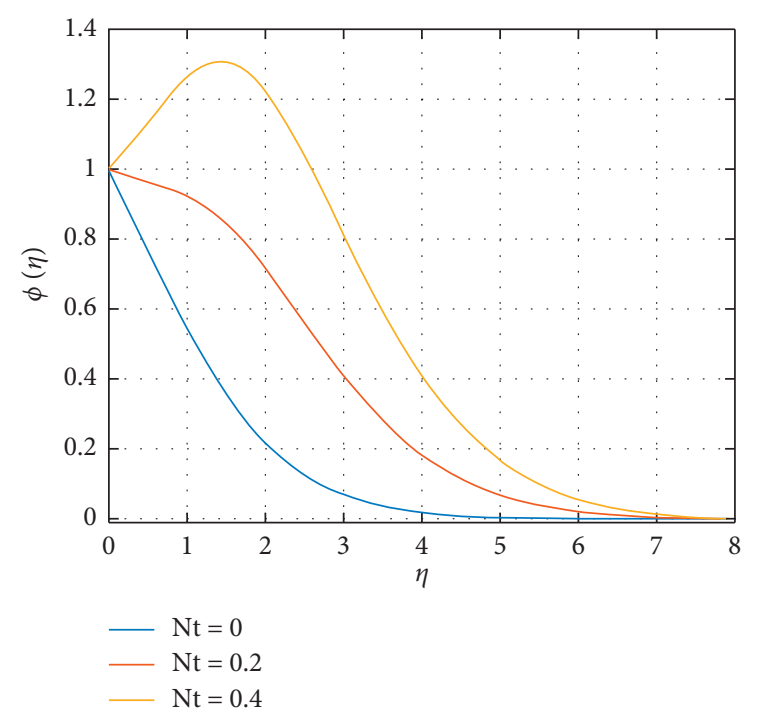

Figure 7: Plot of $\phi(\eta)$ for variation in $N_{t}$ when $\mathrm{Pr}=\mathrm{Le}=\mathrm{Gr}=\mathrm{Sc}=\mathrm{Pe}=1.0, c=0.5$, and $N_{r}=N_{b}=0.1$.

volume fraction boundary layer remarkably. However, thermophoresis impact is crucial in mixed convection of nanofluids, and there are other parameters that may be assumed and have impacts. Such influences incorporate decline in essential kinematic viscosity of nanofluid due to the existence of nanoparticles and density alteration cause of variant volume fraction with heat capacity of the nanofluid. Contrarily, it is presented that the separation element for ordinary nanofluids is positive, and density alteration by cause of modified volume fraction of nanoparticles, known as particulate buoyancy force, assists nanofluid to have higher convective heat transmission.

Figures 8 and 9 show the significant changes in the temperature, and nanoparticles' concentration graph occurred due to changes in Brownian parameter $N_{b}$; when

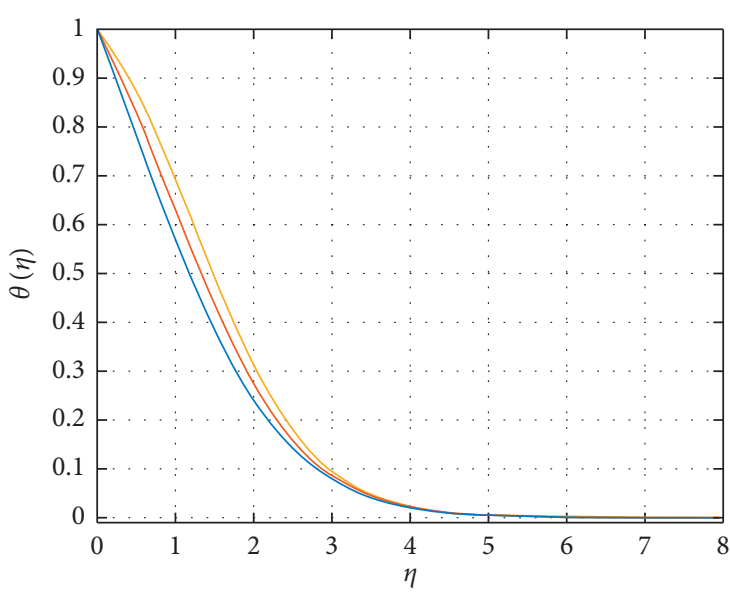

$\begin{aligned} \mathrm{Nb} & =0.1 \\ \mathrm{Nb} & =0.8 \\ \mathrm{Nb} & =1.5\end{aligned}$

Figure 8: Plot of $\theta(\eta)$ for variation in $N_{b}$ when $\mathrm{Pr}=\mathrm{Le}=\mathrm{Gr}=\mathrm{Sc}=\mathrm{Pe}=1.0, c=0.5$, and $N_{t}=N_{r}=0.1$.

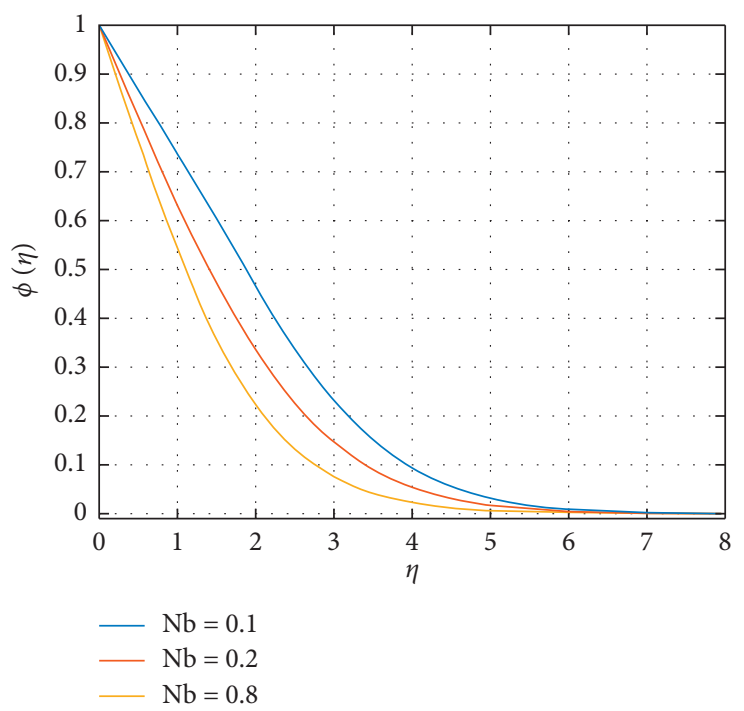

Figure 9: Plot of $\phi(\eta)$ for variation in $N_{b}$ when $\mathrm{Pr}=\mathrm{Le}=\mathrm{Gr}=\mathrm{Sc}=\mathrm{Pe}=1.0, c=0.5$, and $N_{t}=N_{r}=0.1$.

there exists a temperature gradient in the flow region of the propagation, tiny particles scatter rapidly in hotter area and slower in colder domain. The combined impact of the differential distribution of the particles is their movement from hotter to colder region of the fluid area. That is, in the existence of a temperature gradient, particles migrate on the mean average rate in opposition to this gradient. This average motion is called thermophoresis. The fluid temperature increases when $N_{b}$ increases due to the rapid increase in heat transfer but concentration profile decreases when $N_{b}$ increases because the mass transfer decreases due to the enhancement in $N_{b}$. In Figures 10 and 11, the effect of thermophoresis and Brownian parameter on microorganisms' density $w(\eta)$ is evaluated. The density of microorganisms increases 


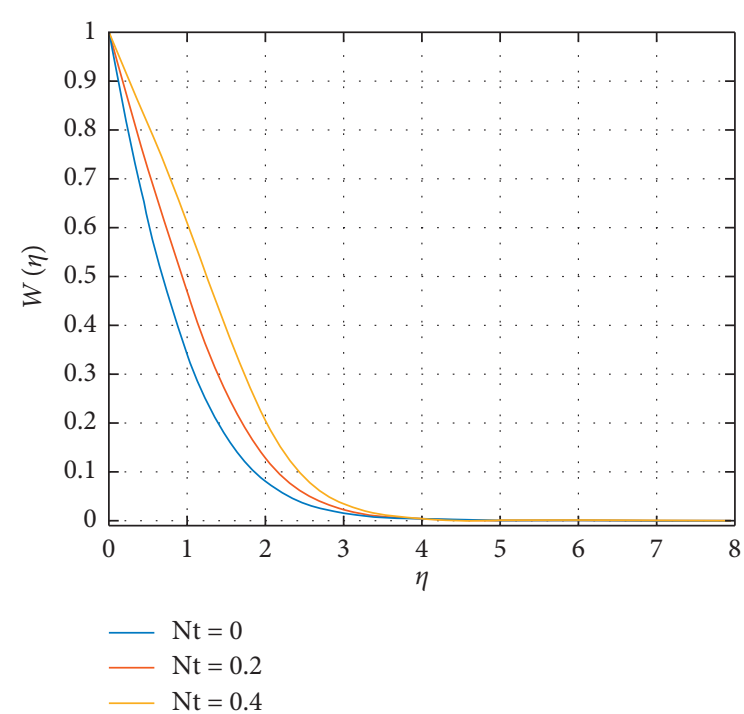

Figure 10: Plot of $w(\eta)$ for variation in $N_{t}$ when $\operatorname{Pr}=\mathrm{Gr}=\mathrm{Le}=\mathrm{Sc}=\mathrm{Pe}=1.0, c=0.5$, and $N_{r}=N_{b}=0.1$.

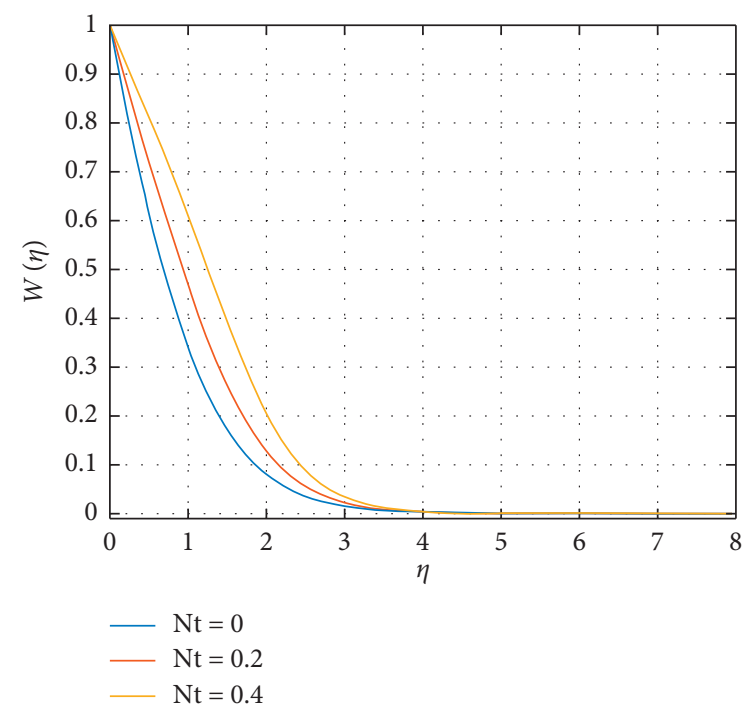

Figure 11: Plot of $w(\eta)$ for variation in $N_{b}$ when $\operatorname{Pr}=\mathrm{Gr}=\mathrm{Le}=\mathrm{Sc}=\mathrm{Pe}=1.0, c=0.5$, and $N_{r}=N_{t}=0.1$.

continuously as $N_{t}$ increases; however, it decreases as $N_{b}$ increases. This behavior indicates that the addition of nanoparticles with bioconvection can affect the distribution and classification microorganisms' density in the fluid to a big scale. Figures 12 and 13, present the impact of Peclet $(\mathrm{Pe})$ and Schmidt number $(\mathrm{Sc})$ on motile microorganisms. Pe is the remarkable fact to investigate the microorganisms swimming in the fluid, and it can be described as the ratio between maximum cell swimming speed and diffusion of microorganisms. Diffusion happens when a material migrates from a region of higher concentration to lower concentration. It explores the tendency of the mass in the fluid. It is established that diffusivity of microorganisms is declined when an expansion is occur inPe. As a consequence, the

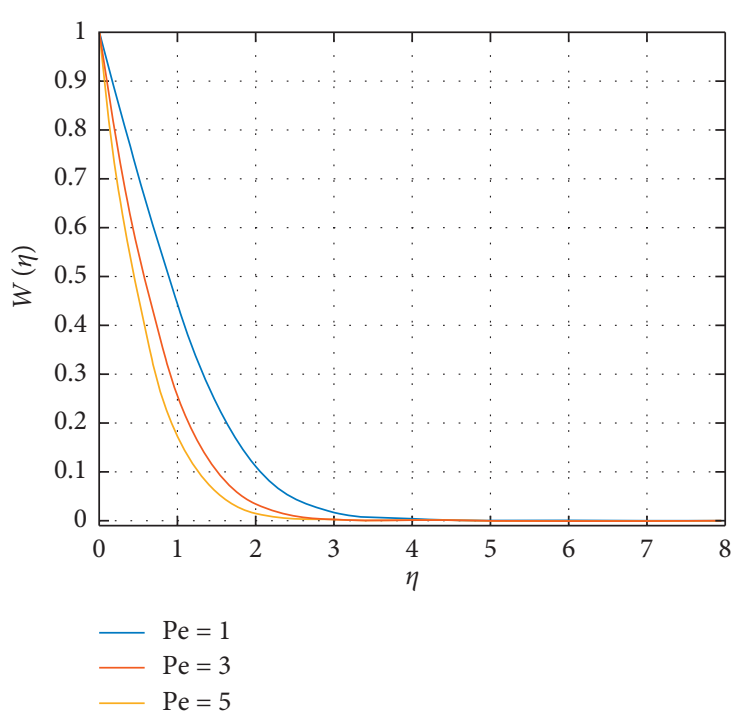

Figure 12: Plot of $w(\eta)$ for variation in $\mathrm{Pe}$ when $\operatorname{Pr}=\mathrm{Gr}=\mathrm{Le}=\mathrm{Sc}=1.0, c=0.5$, and $N_{r}=N_{t}=N_{b}=0.1$.

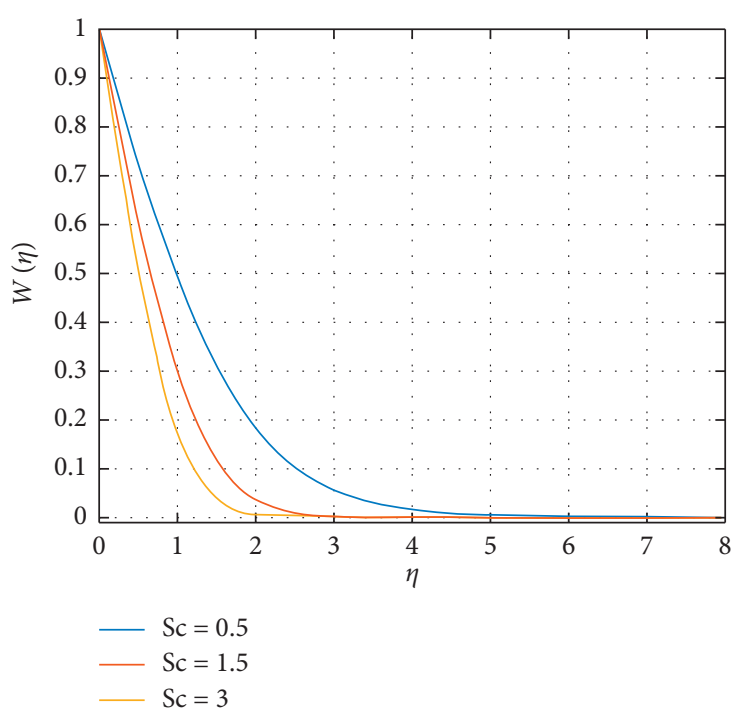

Figure 13: Plot of $w(\eta)$ for variation in $\mathrm{Pe}$ when $\operatorname{Pr}=\mathrm{Gr}=\mathrm{Le}=\mathrm{Pe}=1.0, c=0.5$, and $N_{r}=N_{t}=N_{b}=0.1$.

microrotation diffusion deduces. Hence, the thickness of boundary layer reduces for motile microorganisms.

Table 1 expresses the ranges of physical parameters for a stable solution. Table 2 describes the impact of Prandtl number and bouncy parameter on local skin friction coefficients along $x$ - and $y$-axes. It is clear that these two parameters have considerable effect on local skin friction. Enlarging both $N_{r}$ and Pr leads to decline in $C_{f x}$ and $C_{f x}$. In Table 3, the impact of $N_{t}$ and $N_{b}$ on Sherwood number and local Nusselt number is presented. When $N_{b}$ increases, the Nusselt number reduces, but the Sherwood number increases. In Table 4, the impact of Pe and Sc on microorganisms density is discussed. The quantitative data indicates an increase in the density of microorganisms for higher values of both $\mathrm{Pe}$ and. Sc 
TABLE 1: The range of distinct parameters for a stable solution.

\begin{tabular}{lccccc}
\hline $\mathrm{Pe}$ & $\mathrm{Le}$ & $N_{b}$ & $N_{t}$ & $\operatorname{Pr}$ & $\mathrm{Gr}$ \\
\hline $\mathbf{0 - 2 5}$ & 1.0 & 0.1 & 0.1 & 1.0 & 1.0 \\
1.0 & $\mathbf{0 . 1 - 2 0}$ & 0.1 & 0.1 & 1.0 & 1.0 \\
1.0 & 1.0 & $\mathbf{0 . 1 - 2}$ & 0.1 & 1.0 & 1.0 \\
1.0 & 1.0 & 0.1 & $\mathbf{0 . 1 - 2}$ & 1.0 & 1.0 \\
1.0 & 1.0 & 0.1 & 0.1 & $\mathbf{1 - 2 0}$ & 1.0 \\
1.0 & 1.0 & 0.1 & 0.1 & 1.0 & $\mathbf{1 - 1 5}$ \\
\hline
\end{tabular}

The bold values indicate the ranges of physical parameters for stable solution.

TABle 2: Local skin friction $\operatorname{Re}_{x}^{1 / 2} C_{f x}=f^{\prime \prime}(0)$ and $\operatorname{Re}_{y}^{1 / 2} C_{f y}=$ $h^{\prime \prime}(0)$ along $x$ - and $y$-axes, respectively, corresponding to various values of $N_{r}$ and Pr.

\begin{tabular}{cccccc}
\hline$N_{r}$ & $f^{\prime \prime}(0)$ & $h^{\prime \prime}(0)$ & $\operatorname{Pr}$ & $f^{\prime \prime}(0)$ & $h^{\prime \prime}(0)$ \\
\hline 0.1 & 0.7023 & 0.7439 & 1 & 0.7023 & 07439 \\
0.3 & 0.5556 & 0.5845 & 5 & 0.5743 & 0.5934 \\
0.4 & 0.4765 & 0.4980 & 1 & 0.5072 & 0.5200 \\
\hline
\end{tabular}

TABLE 3: Local Nusselt number in comparison with local Sherwood number $\operatorname{Re}_{x}^{(-1 / 2)} \mathrm{Nu}_{x}=\operatorname{Re}_{y}^{(-1 / 2)} \mathrm{Nu}_{y}=-\theta^{\prime}(0)$ and $\mathrm{Re}_{x}^{-(1 / 2)} \mathrm{Sh}_{x}=$ $\operatorname{Re}_{y}^{-(1 / 2)} \operatorname{Sh}_{y}=-\phi^{\prime}(0)$, for different $N_{t}$ and $N_{b}$.

\begin{tabular}{cccccc}
\hline$N_{b}$ & $-\theta^{\prime}(0)$ & $-\phi^{\prime}(0)$ & $N_{t}$ & $f^{\prime \prime}(0)$ & $h^{\prime \prime}(0)$ \\
\hline 0.1 & 0.4301 & 0.2703 & 0.1 & 0.4301 & 0.2703 \\
0.8 & 0.3106 & 0.4233 & 0.3 & 0.3948 & -0.0738 \\
1.5 & 0.2118 & 0.4914 & 0.4 & 0.3782 & -0.2223 \\
\hline
\end{tabular}

TABLE 4: Density of motile microorganisms $\operatorname{Re}_{x}^{-(1 / 2)} Q_{n x}=-w(0)$, for various values of $\mathrm{Pe}$ and Sc.

\begin{tabular}{lccc}
\hline $\mathrm{Pe}$ & $-w^{\prime}(0)$ & $\mathrm{Sc}$ & $-w^{\prime}(0)$ \\
\hline 1 & 0.7179 & 0.5 & 0.5727 \\
3 & 1.1232 & 1.5 & 0.8206 \\
5 & 1.5751 & 3 & 1.0311 \\
\hline
\end{tabular}

\section{Conclusions}

A detailed analysis of boundary layer steady flow with nanoparticles and gyrotactic microorganisms is presented. The transformed boundary value problem is deal numerically with MATLAB bvp4c ODEs solver. The influence of different values of parameters $N_{b}, N_{r}, N_{b}$, and Le on the fluid velocity, concentration of nanoparticles, temperature, and motile microorganism density profiles are explained qualitatively. Some important points obtained from the results are as follows:

(i) The flow enhances in both directions along $x$ - and $y$-axes as $N_{b}$ and Le increase.

(ii) The velocity profile of fluid reduces as the values of $N_{t}$ and $N_{r}$ enlarge.

(iii) Temperature of the fluid increases as the parameters $N_{b}, N_{t}$, and $N_{r}$ increase but decrease as Le increases.

(iv) The nanoparticles volume fraction profile decreases for high values of $N_{b}$ and Le. The same trend of nanoparticles profile is observed for $N_{t}$ and $N_{r}$.

(v) The microorganisms' density profile decreases for increasing values of $N_{b}, \mathrm{Pe}$, and Sc.

\section{Nomenclature}

Pr:

Sc:

C:

T:

$C_{w}$ :

$T_{w}$ :

$\mathrm{C}_{\infty}$ :

$T_{\infty}$ :

$D_{B}$ :

$u, v$ :

$D_{T}$ :

v:

$D_{n}:$

$v$ :

Ec:

$W_{c}$ :

$g:$

$x, y, z$ :

$\mathrm{Gr}$ :

$\Delta N$ :

Le:

$\theta(\eta)$

$N$ :

$\phi(\eta)$ :

$N_{b}$ :

$\mathrm{Re}_{x}$ :

$f, h, \theta, \phi, w$ :

$v:$

Pe:

$\rho_{f}:$

$N_{t}$ :

$p_{o}$ :

$p:$

Sh:

$C_{f}$ :

$R_{b}$ :

$N_{r}$ :
Prandtl number

Bioconvection schmidt number

Nanofluid volume fraction $\left[\mathrm{kmolm}^{-3}\right]$

Boundary layer temperature $[\mathrm{K}]$

Wall nanoparticle volume fraction

Wall temperature [K]

Ambient nanoparticle volume fraction

Free stream temperature $[K]$

Brownian diffusion coefficient

Velocity components $\left[\mathrm{ms}^{-1}\right]$

Thermophoretic diffusion coefficient

Mean swimming velocity of microorganisms $\left[\mathrm{ms}^{-1}\right]$

Microorganisms' diffusivity

Velocity vector

Eckert number

Maximum speed of swimming cell

Gravitational constant $\left[\mathrm{ms}^{-2}\right]$

Rectangular coordinates

Grash of number

Characteristic motile microorganisms' density difference

Lewis number

Dimensionless temperature

Microorganisms' concentration

Dimensionless nanoparticle volume fraction

Brownian motion parameter

Thermophoresis diffusion coefficient

Dependent variables

Kinematic viscosity $\left[\mathrm{m}^{2} \mathrm{~s}^{-1}\right]$

Peclet number

Nanofluid density $\left[\mathrm{kgm}^{-3}\right]$

Thermophoresis parameter

Stagnation pressure

Pressure $[\mathrm{kPa}]$

Sherwood number

Skin friction coefficient

Bio convection Rayleigh number

Buoyancy parameter.

\section{Data Availability}

The data used to support the findings of this study are included within the article.

\section{Conflicts of Interest}

The authors declare that they have no conflicts of interest. 


\section{Acknowledgments}

This work was supported by the National Natural Science Foundation of China (Approval no. 12062018) and the Natural Science Foundation of Inner Mongolia (Approval nos. 2018LH01016 and 2020MS01015).

\section{References}

[1] S. U. S. Choi and J. A. Eastman, "Enhancing thermal conductivity of fluids with nanoparticles," in Proceedings of the 1995 International Mechanical Engineering Congress and Exhibition, San Francisco, CA, USA, October 1995.

[2] S. K. Das, S. U. Choi, W. Yu, and T. Pardeep, Nanofluids: Science and Technology, John Wiley \& Sons, New York, NY, USA, 2007.

[3] K. Jahani, M. Mohammadi, M. B. Shafi, and Z. Shiee, "Promising technology for electronic cooling: nanoflidic micro pulsating heat pipes," Journal of Electronic Packaging, vol. 135 , no. $2,2013$.

[4] S. A. Khan, H. Waqas, S. M. R. S. Naqvi, M. Alghamdi, and Q. Al-Mdallal, "Cattaneo-Christov double diffusions theories with bio-convection in nanofluid flow to enhance the efficiency of nanoparticles diffusion," Case Studies in Thermal Engineering, vol. 26, Article ID 101017, 2021.

[5] A. Raees, U. Farooq, M. Hussain, W. A. Khan, and F. B. Farooq, "Non-similar mixed convection analysis for magnetic flow of second-grade nanofluid over a vertically stretching sheet," Communications in Theoretical Physics, vol. 73, no. 6, 2021.

[6] U. Farooq, M. Hussain, M. A. Ijaz, W. A. Khan, and F. B. Farooq, "Impact of non-similar modeling on DarcyForchheimer-Brinkman model for forced convection of Casson nano-fluid in non-Darcy porous media," International Communications in Heat and Mass Transfer, vol. 125, pp. 1735-1933, Article ID 105312, 2021.

[7] J. Buongiorno, "Convective transport in nanofluids," Journal of Heat Transfer, vol. 128, no. 3, pp. 240-250, 2006.

[8] A. V. Kuznetsov and D. A. Nield, "Natural convective boundary-layer flow of a nanofluid past a vertical plate," International Journal of Thermal Sciences, vol. 49, no. 2, pp. 243-247, 2010.

[9] N. Bachok, A. Ishak, R. Nazar, and I. Pop, "Flow and heat transfer at a general three-dimensional stagnation point in a nanofluid," Physica B: Condensed Matter, vol. 405, no. 24, pp. 4914-4918, 2010.

[10] N. Bachok, A. Ishak, and I. Pop, "Stagnation-point flow over a stretching/shrinking sheet in a nanofluid," Nanoscale Research Letters, vol. 6, 2011.

[11] A. Alsaedi, M. Awais, and T. Hayat, "Effects of heat generation/absorption on stagnation point flow of nanofluid over a surface with convective boundary conditions," Communications in Nonlinear Science and Numerical Simulation, vol. 17, no. 11, pp. 4210-4223, 2012.

[12] R. Razzaq, U. Farooq, J. Cui, and T. Muhammad, "Nonsimilar solution for magnetized flow of Maxwell nanofluid over an exponentially stretching surface," Mathematical Problems in Engineering, vol. 10, Article ID 5539542, , 2021.

[13] J. R. Platt, "“Bioconvection patterns" in cultures of freeswimming organisms," Science, vol. 133, no. 3466, pp. 1766-1767, 1961.

[14] S. Milton and H. Plesset, "Bioconvection patterns in swimming microorganism cultures as an example of Rayleightaylor instability," Nature London, vol. 248, pp. 441-443, 1974.
[15] S. Childress, M. Levandowsky, and E. A. Spiegel, "Pattern formation in a suspension of swimming microorganisms: equations and stability theory," Journal of Fluid Mechanics, vol. 69, no. 3, pp. 591-613, 1975.

[16] M. Levandowsky, W. S. Childress, E. A. Spiegel, and S. H. Hutner, "A mathematical model of pattern formation by swimming microorganisms," The Journal of Protozoology, vol. 22, no. 2, pp. 296-306, 1975.

[17] T. J. Pedley, N. A. Hill, and J. O. Kessler, "The growth of bioconvection patterns in a uniform suspension of gyrotactic micro-organisms," Journal of Fluid Mechanics, vol. 195, pp. 223-237, 1988.

[18] T. J. Pedley and J. O. Kessler, "Hydrodynamic phenomena in suspensions of swimming microorganisms," Annual Review of Fluid Mechanics, vol. 24, no. 1, pp. 313-358, 1992.

[19] M. A. Bees, "Non-linear pattern generation in suspensions of swimming micro-organisms," $\mathrm{PhD}$ Thesis, University of Leeds, Leeds, UK, 1996.

[20] M. A. Bees and N. A. Hill, "Linear bioconvection in a suspension of randomly swimming, gyrotactic micro-organisms," Physics of Fluids, vol. 10, no. 8, pp. 1864-1881, 1998.

[21] S. Ghorai and N. A. Hill, "Development and stability of gyrotactic plumes in bioconvection," Journal of Fluid Mechanics, vol. 400, pp. 1-31, 1999.

[22] S. Ghorai and N. A. Hill, "Periodic arrays of gyrotactic plumes in bioconvection," Physics of Fluids, vol. 12, no. 1, pp. 5-22, 2000.

[23] S. Ghorai and N. A. Hill, "Wavelengths of gyrotactic plumes in bioconvection," Bulletin of Mathematical Biology, vol. 62, no. 3, pp. 429-450, 2000.

[24] S. Ghorai and N. A. Hill, "Axisymmetric bioconvection in a cylinder," Journal of Theoretical Biology, vol. 219, no. 2, pp. 137-152, 2002.

[25] A. V. Kuznestov, "Thermo-Bioconvection in a suspension of oxytactic bacteria," International Communications in Heat and Mass Transfer, vol. 32, pp. 991-999, 2005.

[26] A. V. Kuznestov, "The onset of bioconvection in a suspension of gyrotactic microorganisms in a fluid layer of finite depth heated from below," International Communications in Heat and Mass Transfer, vol. 32, pp. 574-582, 2005.

[27] A. V. Kuznestov, "Investigation of the onset of thermoBioconvection in a suspension of oxytactic microorganisms in a shallow fluid layer heated from below," Theoretical and Computational Fluid Dynamics, vol. 19, pp. 287-299, 2005.

[28] P. Geng and A. V. Kuznetsov, "Effect of small solid particles on the development of bioconvection plumes," International Communications in Heat and Mass Transfer, vol. 31, no. 5, pp. 629-638, 2004.

[29] P. Geng and A. V. Kuznetsov, "Settling of bidispersed small solid particles in a dilute suspension containing gyrotactic micro-organisms," International Journal of Engineering Science, vol. 43, no. 11-12, pp. 992-1010, 2005.

[30] P. Geng and A. V. Kuznestov, "Introducing the concept of effective diffusivity to evaluate the effect of bioconvection on small solid particles," International Journal of Transport Phenomena, vol. 7, pp. 321-338, 2005.

[31] K. Zaimi, A. Ishak, and I. Pop, "Stagnation-point flow toward stretching/shrinking sheet in a nanofluid containing both nanoparticles and gyrotactic microorganisms," Journal of Heat Transfer, vol. 136, Article ID 41705, 2014.

[32] L. Howarth, "The boundary layer in three dimensional flow.part II. The flow near a stagnation point," The London, Edinburgh, and Dublin Philosophical Magazine and Journal of Science, vol. 42, no. 335, pp. 1433-1440, 1951. 
[33] A. Davey and D. Schofield, "Three-dimensional flow near a two-dimensional stagnation point," Journal of Fluid Mechanics, vol. 28, no. 1, pp. 149-151, 1967.

[34] G. Poots, "Laminar free convection near the lower stagnation point on an isothermal curved surface," International Journal of Heat and Mass Transfer, vol. 7, no. 8, pp. 863-874, 1964.

[35] A. Slaouti, H. S. Takhar, and G. Nath, "Unsteady free convection flow in the stagnation-point region of a three-dimensional body," International Journal of Heat and Mass Transfer, vol. 41, no. 22, pp. 3397-3408, 1998.

[36] S. D. Harris, D. B. Ingham, and I. Pop, "Transient free convection near the lower stagnation point of a cylindrical surface subjected to a sudden change in surface temperature," International Communications in Heat and Mass Transfer, vol. 27, no. 8, pp. 1091-1100, 2000.

[37] H. Xu, S.-J. Liao, and I. Pop, "Series solutions of unsteady free convection flow in the stagnation-point region of a threedimensional body," International Journal of Thermal Sciences, vol. 47, no. 5, pp. 600-608, 2008.

[38] A. K. Singh, N. P. Singh, A. K. Singh, M. Gupta, and P. Agnihotri, "Unsteady free convection and mass transfer flowin the stagnation-point region of a three-dimensional body: use of homotopy analysis method," Computational Thermal Sciences, vol. 2, no. 6, pp. 501-514, 2010.

[39] M. A. Admon, S. Shafie, and I. Pop, "Unsteady free convection flow near the stagnation point of a three-dimensional body," Journal of Applied Sciences, vol. 11, no. 8, pp. 1441-1444, 2011.

[40] U. Farooq and H. Xu, "Free convection nanofluid flow in the stagnation-point region of a three-dimensional body," The Scientific World Journal, vol. 2014, p. 14, Article ID 158269, 2014. 